\title{
Educación como disciplina y como objeto de estudio: aportes para un debate
}

\section{Education as a discipline and as an object of study: contributions to the debate}

\section{María Loreto Mora-Olate ${ }^{1}$}

Universidad Autónoma de Chile, Chile / Colegio Concepción de Chillán, Chile

maria.mora@uautonoma.cl

ORCID: 0000-0002-7631-9179

Citar como: Mora-Olate, M. (2020). Educación como disciplina y como objeto de estudio: aportes para un debate. Desde el Sur, 12(1), pp. 201-211.

\section{RESUMEN}

El ensayo tiene por objetivo dar cuenta de un debate que parece persistir acerca del rango científico de la educación o de si es más correcto hablar de «ciencia» o «ciencias de la educación». Mediante una revisión bibliográfica se intenta responder a la pregunta: ¿en qué se reconoce la educación? Se concluye que, debido a la complejidad del fenómeno educativo, hablar de «ciencias de la educación» no constituiría subsidiariedad, sino más bien una sensata y necesaria interdependencia con las demás disciplinas, que permiten estudiar la educación en su real dimensión social, histórica, biológica y psicológica.

\section{PALABRAS CLAVE}

Educación, ciencias de la educación, disciplina

1 Doctora en Educación, magíster y licenciada, y profesora de Castellano y Comunicación Social por la Universidad del Bío-Bío (Chile). Máster en Educación, Asesoramiento Educativo Familiar, Centro Universitario Villanueva, Universidad Complutense de Madrid (MadridSantiago de Chile). Becaria de la Comisión Nacional de Investigación Científica y Tecnológica (Conicyt, 2017-2020). Durante 15 años se ha dedicado a la formación del profesorado en la Facultad de Educación y Humanidades en la Universidad del Bío-Bío. Ha publicado en diversas revistas indexadas en Venezuela, Cuba, México, Uruguay y Chile, en la línea de educación y diversidad cultural migrante y, a su vez, se ha desempeñado como par evaluador. Se encuentra adscrita como investigadora en el grupo de Investigación Familia, Escuela y Sociedad (FESOC) de la Universidad del Bío-Bío, y en el grupo de Investigación Literatura y Escuela (LyE) de la Universidad Autónoma de Chile. Desde marzo de 2020, se desempeña como vicerrectora académica en el Colegio Concepción de Chillán, región de Ñuble, Chile. 


\section{ABSTRACT}

The aim of this essay is to give an account of a debate that still seems to be very much alive, concerning the science of education and if it is more correct to speak of "science" or "educational sciences". Through a bibliographic review, this study attempts to provide an answer to the question: how is education seen? It is concluded that, given the complexity of the educational phenomenon, to speak of "educational sciences" would not constitute subsidiarity, but rather a rational and necessary interdependence with other disciplines, enabling the study of education in its true social, historical, biological and psychological dimensions.

\section{KEYWORDS}

Education, educational sciences, discipline

\section{Introducción}

El concepto de educación se ha usado en un sentido muy amplio y ha variado conforme el tiempo histórico, como muy bien lo sintetiza Durkheim:

en las ciudades griegas y latinas, la educación enseñaba al individuo a subordinarse ciegamente a la colectividad; en Atenas, se trataba de formar mentes delicadas, cautas, sutiles, amantes de la mesura y de la armonía; en Roma, se deseaba ante todo que los niños se hiciesen hombres de acción, entusiastas de la gloria militar. En el Medioevo, la educación era ante todo cristiana; en el trascurso del Renacimiento, adopta un carácter más laico y más literario; hoy en día, la ciencia tiene tendencia a ocupar en la educación el puesto que el arte tenía antaño $(1975$, p. 44).

A su vez, la historia de la educación es también la historia de la cultura; existe una significación común que cruza el recorrido diacrónico, entendiendo como educación la influencia intencional y sistemática por parte de los adultos sobre el ser juvenil con el propósito de formarlo o desarrollarlo, a la vez que se conserva y transmite la existencia colectiva (Durkheim, 1975 y Luzuriaga, 1971). Por su parte, Runge y Muñoz (2012) relevan a la educación como «la praxis de los humanos adultos para con los que están en crecimiento, o para los que necesitan de educación» (p. 80). Se observa entonces que en estas conceptualizaciones subyace el acto de enseñar como objeto de la educación.

En el siguiente ensayo se presentan los aportes de autores que se han considerado como significativos en relación con este ejercicio reflexivo en torno a la educación como disciplina y objeto de estudio. El debate parece 
persistir acerca del rango científico de la educación, o si es más correcto hablar de «ciencia» o «ciencias de la educación». Esta situación también conlleva implicancias en la forma en que se entiende la investigación en el campo de la educación. Al mismo tiempo, la lectura de diversos autores remite a la necesidad de buscar la precisión conceptual y conlleva a una reflexión de dichos conceptos, lo que comprende su contexto de producción y su resignificación actual.

\section{La educación como fenómeno}

La educación es un fenómeno sociocultural de carácter universal, que implica en sí un acto de transmisión cultural de viejas a nuevas generaciones. Se subraya «el carácter más general y fundamental de una cultura: que debe ser aprendida; o sea, transmitida en alguna forma» (Abbagnano y Visalberghi, 1992, p. 11). Dicha transmisión es la educación, un fenómeno determinante para el desarrollo individual y social, y desentrañar sus mecanismos y sus leyes "supone acceder a niveles explicativos integradores de las distintas dimensiones de la realidad, física, biológica, psicológica y social, de los que todavía nos encontramos muy alejados» (De la Torre, 2006, p. 16).

Freire revela el significado epistemológico profundo de la educación: la relación, el diálogo entre profesor y alumno, y no solo eso, sino que se otorga dignidad al aprendiz (Andreola, en Gadotti, 2003, p. 151). En esa misma línea, Maturana y Dávila (2006) la conciben como «un fenómeno de transformación en la convivencia, en un ámbito donde el educando no aprende una temática, sino un vivir y un convivir» (p. 32), con los seres humanos y la naturaleza. De acuerdo con lo anterior, según Maturana, la tarea de la educación consiste en «crear un espacio relacional en el que nuestros niños puedan crecer para vivir en el presente, en cualquier presente, conscientes del futuro posible o deseado, pero no alineado en ninguna descripción de él» (Maturana en López Melero et al., 2003, p. 12).

En estos planteamientos subyace una idea de educación desde la racionalidad dialéctica, que, sumada a la racionalidad comunicativa, interpelan a la educación a «abordar de forma más completa los aspectos educativos al incluir la problemática de los contrarios, que implica la aceptación del conflicto mediante pretensiones de validez, dada en la búsqueda de una comprensión intersubjetiva en la que los miembros capaces de lenguaje y acción se presentan en igualdad de condiciones» (Ferrada, 2001, p. 12).

\section{La educación como disciplina y objeto de estudio}

Es posible reconocer un campo denominado educación y resulta legítimo que existan diferentes saberes en ese campo, pero hay una disciplina propia llamada educación, cuyo objeto de estudio lo constituye el acto 
universal de enseñar, la pedagogía, que en sí entraña una complejidad por la cual confluyen diversos saberes, como la biología, la psicología, la sociología, la antropología, la filosofía, entre otros. Rojas (2006) apunta al tratamiento conceptual de la pedagogía en su movimiento entre dos tratamientos, «por una parte el estatuto de ciencia unificadora, y por otra el de una disciplina más dentro de un campo de conocimiento cuyo objeto de estudio es la educación» (p. 23).

Para Luzuriaga (1971), la educación y la pedagogía «están en la relación de la práctica y la teoría, de la realidad y la idealidad, de la experiencia y el pensamiento, pero no como entidades independientes, sino fundidas en una unidad indivisible, como el anverso y el reverso de una moneda» (p. 12). Así, la complejidad del objeto educativo implica la considerar el aporte de diferentes disciplinas; de lo contrario, «el análisis de lo educativo será forzosamente imparcial e incompleto» (Martínez-Otero, 2004, p. 35).

Runge y Muñoz (2012) nos recuerdan que antes de la modernidad la educación no fue un tema en torno al cual se pensara, es decir, se desarrollaba sin un método sistemático. Esto cambia cuando emerge «la pedagogía como reflexión sobre la educación, pues los seres humanos se ven enfrentados a cuestiones como: ¿cuál es la forma de vida que se le va a presentar de un modo sistemático a las nuevas generaciones?, ¿qué se les va a presentar?, ¿cómo se les va a educar, para qué se les va a educar?» (p. 90).

En vínculo estrecho con los conceptos de educación y pedagogía la bibliografía da cuenta de un antiguo debate en relación, por un lado, con el carácter autónomo científico de la educación (ciencia de la educación) $y$, por otro, si esta constituye una disciplina dependiente de otras (ciencias de la educación), las cuales «se complementan, fortalecen y enriquecen en una suerte de mapa multidisciplinar» (Martínez-Otero, 2004). El surgimiento de dichas discusiones datan de principios del siglo XX, producto de los intensos debates entre los «fervientes defensores y partidarios de una ciencia de la educación y aquellos que veían en la complejidad del concepto de educación el sentido del plural ciencias» (Zambrano, 2006, p. 594). Aquellos debates transitaron en la búsqueda de otorgarle a la educación un estatus de ciencia experimental, cuyo propósito radicaría en «el estudio del fenómeno social llamado educación, que hiciera posible, entre otros propósitos, su control y planeación» (Zuluaga, Echeverry, Martínez, Restrepo y Quiceno, 1988, p. 95); las disciplinas constitutivas eran la pedagogía, la didáctica, la filosofía, la psicología, la antropología, entre otras. En síntesis, los primeros, desde la tradición alemana, eran partidiarios de la denominación en singular y consideraban a la pedagogía como la ciencia de la educación, que «prescribía el conjunto de técnicas 
necesarias en el desarrollo de la inteligencia, [mientras que] los segundos, desde la corriente francesa y anglosajona, el acto de educar no se limitaba a tal desarrollo, sino que abarcaba todo el conjunto de instituciones, las prácticas, los modos y las formas como la sociedad adulta educaba a las generaciones más jóvenes» (Zambrano, 2006, p. 594). García Carrasco y García del Dujo (1996) plantean que «cuando hablamos de educación y lo hacemos con preocupaciones científicas, tenemos que partir de un punto de vista inicial insoslayable. Los acontecimientos educativos son hechos y acontecimientos del mundo, pero ello no implica que sean reductibles a meros fenómenos físicos, aunque altamente complejos» (p. 39). Ello evidencia que en el debate acerca del estatus científico de la educación incluye la influencia paradigmática del pensamiento positivista, que, a juicio de Medina Rubio (en Martínez-Otero, 2004), obedece a una «actitud reduccionista que pretende constituirse en el único saber valioso posible [...] cada ciencia tiene su propio sistema de enunciados y su peculiar forma de acceder a la realidad en la que incide» (p. 37).

Es preciso señalar que la práctica de la educación es muy anterior al pensamiento pedagógico. El pensamiento pedagógico surge con la reflexión sobre la práctica de la educación, como necesidad de sistematizarla y organizarla en función de determinados fines y objetivos (Gadotti, 2003, p. 7).

Al respecto, Gadotti (2003) apunta que Comenio, ya en 1657, realizó aquella primigenia sistematización, con su Didáctica magna, bajo la premisa de que «la escuela debe enseñar el conocimiento de las cosas», propone un método pedagógico para enseñar con «rapidez, economía de tiempo y sin fatiga» (Gadotti, 2003, p. 70). En otras palabras, continúa Gadotti (2003), Comenio «hace un intento por crear ciencia de la educación utilizando los mismos métodos de las ciencias físicas» (p. 74). El pensamiento de Kant contrasta con Comenio, al realizar un esfuerzo de precisión en los conceptos de educación, pedagogía y didáctica, ya que el filósofo alemán concibe a la pedagogía como «disciplina académica: adquiere así un corpus teórico más consistente, bajo la forma de un tratado puntual y concreto de corte eminentemente filosófico, con el desarrollo de categorizaciones, fines y principios de la educación» (Rojas, 2006, p. 21). Entonces, la pedagogía, desde la tradición alemana, es concebida como equivalente a ciencia de la educación, en contraposición a las tradiciones francesa y anglosajona, en las que no alcanza dicho estatus científico, «por carecer de un modelo teórico-metodológico que dé sustento al conocimiento que le es propio» (Rojas, 2006, p. 25). Lundgren (1992) confirma la influencia de la filosofía en el desarrollo de la educación como ciencia y señala a la teoría comprensiva de la educación de Herbart «como 
el primer intento profundo de conformar la educación en una disciplina», al considerar, por un lado, «cómo se podría adquirir el conocimiento sobre la selección y la organización del contenido para enseñar, y por otro, cómo se podría obtener el conocimiento sobre la forma en que este debía transmitirse» (Lundgren, 1992, p. 49).

Abbagnano y Visalberghi (1992) afirman que el término pedagogía, que literalmente significa "guía del niño», puede tener un significado más extenso y abarcar, además de la filosofía de la educación, algunas ciencias y sectores de aquellas ciencias indispensables para un control del proceso educativo: la psicología, sobre todo las partes referidas al desarrollo mental, a la formación del carácter y a los modos de aprendizaje; y la sociología, que ha demostrado ser una indispensable ciencia auxiliar para plantear y resolver debidamente los problemas de la educación. Junto a la psicología y a la sociología, se ha desarrollado una técnica o conjunto de técnicas que emergen de la práctica educativa misma: la didáctica. Por lo tanto, dichos autores asignan a la pedagogía las tareas de «coordinar las contribuciones de las diversas ciencias auxiliares y técnicas didácticas, y de impedir que se caiga en recetas fijas, de evitar que se cristalicen los métodos y los valores y, en resumen, de llevar a cabo aquella misión de apertura hacia lo nuevo y lo diverso que tiene en común con la filosofía (Abbagnano y Visalberghi, 1992, p. 15).

Para Nóvoa (2009), el año 1920, con la publicación del libro Transformemos la escuela de Adolphe Ferrière, es un hito simbólico de la modernidad escolar y pedagógica, porque se trata de un decanto de los avances que se desarrollaban desde 1870 en el pensamiento pedagógico. La idea que resume el movimiento de la Escuela Nueva y la pedagogía moderna es el concepto de «educación integral», que, a juicio de este autor, causó que la «escuela se haya desviado muchas veces de las tareas de enseñanza y de aprendizaje para implicarse en tareas sociales» (Nóvoa, 2009, p. 187). Si bien Nóvoa entronca con Delors (1996) en la idea de que «todos los que estén investidos de alguna responsabilidad presten atención a los objetivos de la educación» (p. 8), él desapega a la escuela de la idea de educación ética y comunitaria.

En la otra vereda, la teoría del conocimiento que transversaliza la obra de Freire releva el significado epistemológico profundo de la educación: la relación, el diálogo entre profesor y alumno, y no solo eso, sino que le otorga «dignidad al aprendiz» (Gadotti, 2003, p. 149) mientras que está «el corazón en el centro del conocimiento» (Andreola, en Gadotti, 2003, p. 151). La pedagogía freiriana, la pedagogía de la praxis, incluye la idea de pensamiento complejo con su mirada planetaria, ecológica, y transdisciplinaria, donde la escuela asume un rol ciudadano (escuela ciudadana). Se 
ha proyectado a futuro la pedagogía freiriana con un contenido ecológico planetario, en el contexto de lo global y con el desafío interdisciplinario.

Continuando con el debate, de la ciencia o ciencias de la educación, Zuluaga et al. (1988) afirman que la pedagogía «no es que haya dejado de existir, sino que las ciencias de la educación le han impuesto una existencia instrumental que hace del maestro un sujeto que aplica teorías producidas en otros saberes y ciencias» (p. 95). Se desplaza así el concepto de enseñanza por el de educación, lo que produce un enrarecimiento y una subordinación de la pedagogía a las acciones que acaecen en la sala de clases, y resulta despojado el profesorado de pensar la escuela, la sociedad, el Estado y la cultura. Concluyen Zuluaga et. al (1988) que la pedagogía ha quedado así reducida en un saber instrumental, y la enseñanza se ve limitada «a procedimientos operativos que conducen al aprendizaje [...] como una disciplina aplicada que debe esperar los avances de las otras ciencias de educación para poder explicar sus resultados» (p. 96). En esta dirección, entonces, a juicio de Ríos (2006), las ciencias de la educación no son pedagogía, porque «solo se limitan a aportar luz clarificadora. Así, podríamos asemejar la pedagogía a una casa donde las ciencias de la educación llegan con sus aportes hasta el dintel de las puertas y ventanas, sin entrar en la casa, pero fortalecen la mirada del visitante y le preparan su visita» (Ríos, 2006, pp. 18-19). De este modo, es posible encontrarse con planteamientos como los de De la Torre (2006), quien reclama «la construcción de un corpus científico e independiente para la educación, la cual, a su juicio, desarrollará su independencia solo si se investigan los procesos de interacción entre maestro y discípulo mediante metodologías cuantitativas y cualitativas, correspondientes a las ciencias nomotécnicas» (p. 26). La pedagogía sigue enfrentando resistencia para constituirse como un campo de saber conceptual y práctico, ya que «por un lado están los profesionales de otras disciplinas que se resisten a relacionarse con ella en el campo de la educación, que parece ser un campo de todos pero a la vez de nadie, un campo donde todas las disciplinas intervienen con un aire de inconmensurabilidad» (Ríos, 2006, p. 28), y, por otro lado, las políticas educativas en Latinoamérica, que tienen como rasgo común el desplazamiento de la pedagogía y de la labor del profesorado en función técnica. El primer obstáculo objeto de análisis lo constituyen las políticas educativas, que son formuladas de acuerdo con las tendencias del mundo globalizado y deslizan «la mirada de los actores insertos en la realidad, en este caso la comunidad educativa local» (Basto, 2009, p. 72).

Sin embargo, De la Torre (2006) estaría asumiendo la subalternidad de la educación como disciplina, al leer en sus planteamientos cierto espíritu apocalíptico en la advertencia referida a la desaparición de la ciencia 
pedagógica, para lo cual «ella debe construir un cuerpo autónomo de conocimientos investigando empíricamente el funcionamiento de profesores y alumnos en acción, si no queremos que nuestra área de conocimiento no desaparezca en el futuro absorbida por los demás (De la Torre, 2006, p. 28).

Si bien De la Torre (2006) releva el objeto de estudio de la educación, la relación entre profesor-alumno, mediada por la enseñanza (y aprendizaje), al reclamar aquella especificidad de la disciplina, transita en una delgada línea de la contradicción, porque señala que la «futura teoría de la educación deberá elaborarse a partir de esa gran diversidad de fuentes epistemológicas integradas» (p. 26). Se refiere a la tradicional línea psicológica y sociológica en educación, a la cual se sumarían los aportes de la neurociencia, cuyos hallazgos «podrían transformar las estrategias educativas y permitirnos idear programas que optimizarán el aprendizaje de las personas de todas las edades y con las más diversas necesidades» (Blakemore y Frith, 2008, p. 19).

Nuevamente, nos encontramos con la necesidad de un diálogo interdisciplinar, ahora entre las ciencias cerebrales y la educación, porque — siguiendo a Blakemore y Frith (2008) — así como podría ser arriesgado sugerir que las investigaciones en el campo de la educación por sí mismas «no proporcionan, o no pueden proporcionar, el mejor enfoque sobre muchas cuestiones educacionales partiendo de sus propios recursos y pensamiento científico razonado», también las autoras se preguntan cómo la neurociencia puede «inspirar a la educación» y que bien vale preguntarse «cómo las ciencias del cerebro suponen un desafío para las opiniones lógicas sobre la enseñanza y el aprendizaje» (Blakemore y Frith, 2008, pp. 27-28).

\section{Conclusiones}

El transitar de las lecturas referenciadas en el presente ensayo ha permitido observar los diferentes planteamientos que retoman el objeto social relacional de la educación como disciplina y objeto de estudio, y también el carácter sociocultural y dialógico del fenómeno educativo que deriva, por tanto, en un objeto de estudio complejo.

A este respecto, se estima que luchar por la «independencia disciplinaria», en cierta medida, olvida la naturaleza del objeto de la educación, considerando además las actuales demandas educativas que interpelan a una mirada interdisciplinaria, ya que el acto de enseñar involucra dimensiones de la realidad, física, biológica, psicológica y social.

Por eso se comparte aquella idea de que la complejidad del objeto educativo llevaría a la necesidad de tener en cuenta las aportaciones de 
las diferentes disciplinas, porque de lo contrario el análisis de lo educativo sería imparcial e incompleto. Por lo tanto, hablar de «ciencias de la educación» no constituiría subsidiariedad, sino más bien una sensata y necesaria interdependencia con las demás disciplinas, que permiten estudiar la educación en su real dimensión social, histórica, biológica y psicológica. Además, «la separatividad de la escuela con el mundo cotidiano nos remiten al debate entre lo teórico y lo práctico de la pedagogía, cuyos niveles de acción, conocimiento pedagógico y acción pedagógica (Bedoya, 2000; García Carrasco y García del Dujo, 1995) nos invitan a revisitar su objeto de estudio desde una postura crítica» (Mora-Olate, 2020, p. 319). Entonces, no se comparte la visión un tanto cataclística de De la Torre (2006), quien apela a una independencia disciplinaria como condición preventiva frente a una supuesta desaparición de la educación como ciencia.

En consecuencia, y llevando la reflexión al terreno de la investigación, la forma de acceder al objeto de estudio de la educación —es decir, al acto de enseñanza- desafía a desarrollar un pensamiento complejo, con mirada interdisciplinaria, lejos de reduccionismos, que permita indagar en los procesos relacionales construidos entre el sujeto que enseña y el que aprende, sin olvidar que este rasgo relacional conlleva también la idea de contexto, con la aspiración de lograr la comprensión intersubjetiva de los sujetos.

\section{Agradecimientos}

Al programa de Doctorado en Educación en Consorcio de la Universidad del Bío-Bío (Chile) y al financiamiento otorgado por la Comisión Nacional de Investigación Científica y Tecnológica (Conicyt), a través del Programa de Formación de Capital Humano Avanzado, Beca Doctorado Nacional 21170381 (2017).

\section{Contribuciones}

María Loreto Mora-Olate ha participado en la concepción, la redacción y la aprobación de la versión final del artículo.

\section{Fuentes de financiamiento}

Financiamiento otorgado por la Comisión Nacional de Investigación Científica y Tecnológica (Conicyt) a través del Programa de Formación de Capital Humano Avanzado, Beca Doctorado Nacional 21170381 (2017).

\section{Conflictos de intereses}

Ninguno. 


\section{REFERENCIAS BIBLIOGRÁFICAS}

Abbagnano, N. y Visalberghi, A. (1992). Historia de la pedagogía. Madrid: Fondo de Cultura Económica.

Basto, S. (2009). Una reflexión sobre ¿qué es la pedagogía? Los desafíos del maestro para hacer de este saber el fundamento y proyección de su quehacer. Revista UIS Humanidades, 37(1), pp. 69-75 Recuperado de https:// revistas.uis.edu.co/index.php/revistahumanidades/article/view/103/733

Blakemore, S. y Frith, U. (2008). Cómo aprende el cerebro. Las claves para la educación. Barcelona: Ariel.

Bedoya, J. I. (2000). Epistemología y pedagogía. Colombia: ECOE.

Delors, J. (1996). La educación encierra un tesoro. Madrid: Santillana y Unesco.

De la Torre, M. (2006). Los cimientos científicos de una nueva teoría educativa. Tendencias pedagógicas. Recuperado de https://revistas.uam.es/ tendenciaspedagogicas/article/view/1865/1974

Durkheim, É. (1975). La educación, su naturaleza y su papel. En Educación y sociología. Barcelona: Península.

Ferrada, D. (2001). Currículum crítico comunicativo. Barcelona: El Roure Editorial.

Gadotti, M. (2003). Historia de las ideaspedagógicas. Ciudad de México:Siglo XXI Editores. Recuperado de https://books.google.cl/books?id=5xRyyoX_ FUoC\&lpg=PR17\&dq=moacir+gadotti+historia+de+las+ideas+pedagogi cas\&hl=es\&pg=PP1\&redir_esc $=y \# v=$ onepage $\& q \& f=$ false

García Carrasco, J. y García del Dujo, Á. (1995). Epistemología pedagógica I. Revista Interuniversitaria, 7, pp. 5-38. Recuperado de https://revistas.usal. es/index.php/1130-3743/article/view/3055/3089

García Carrasco, J. y García del Dujo, Á. (1996). Teoría de la educación I. Salamanca: Ediciones Universidad de Salamanca.

López Melero, M. et al. (2003). Conversando con Maturana de educación. Málaga: Aljibe.

Lundgren, U. P. (1992). Teoría del curriculum y la escolarización. Madrid: Morata.

Luzuriaga, L. (1971). Historia de la educación y de la pedagogía. Argentina: Losada.

Martínez-Otero, V. (2004). Teoría y práctica de la educación. Madrid: CCS.

Maturana, H. y Dávila, X. (2006). Biología del conocer y biología del amar. Desde la matriz biológica de la existencia humana. Prelac, 2, pp. 30-39. Recuperado de https://unesdoc.unesco.org/ark:/48223/pf000014587 
Mora-Olate, M. L. (2020). Educación, pedagogía y desescolarización. Revista Conrado, 16(72), pp. 314-320. Recuperado de https://conrado.ucf.edu. cu/index.php/conrado/article/view/1252

Nóvoa, A. (2009). Educación 2021: para una historia del futuro. Revista lberoamericana de Educación, 49. Recuperado de https://rieoei.org/historico/ documentos/rie49a07.htm

Ríos, R. (2006). De la pedagogía a las ciencias de la educación: una lectura desde el saber pedagógico colombiano. Revista Educación y Pedagogía, 18(44), pp. 11-31. Recuperado de https://revistas.udea.edu.co/index.php/ revistaeyp/article/view/6069/5475

Rojas Moreno, I. (2006). Presencia de los clásicos en la producción discursiva de pedagogía en la Facultad de Filosofía y Letras, UNAM. Perfiles Educativos, XXVIII(113), pp. 7-37. Recuperado de https://www.redalyc.org/ articulo.oa?id=132/13211302

Runge, A. y Muñoz, D. (2012). Pedagogía y praxis (práctica) educativa o educación. De nuevo: una diferencia necesaria. Revista Latinoamericana de Estudios Educativos, 8(2), pp. 75-96. Recuperado de https://www.redalyc.org/articulo.oa?id=134129257005

Zambrano, A. (2006). Las ciencias de la educación y didáctica: hermenéutica de una relación culturalmente específica. Revista Educere, 10(35), pp. 593-599.

Zuluaga, O. L., Echeverry, A., Martínez, A., Restrepo, S. y Quiceno, H. (1988). Educación y pedagogía: una diferencia necesaria. Revista Educación y Cultura, 10, pp. 94-99. Recuperado de http://www.albertomartinezboom. com/escritos/articulos/2013_Educacion_y_pedagogia_Una_diferencia_ necesaria.pdf 